\title{
USING AUGMENTED REALITY AND MIXED REALITY TO INTERPRET DESIGN CHOICES OF HIGH-PERFORMANCE BUILDINGS
}

\author{
Shenghuan Zhao ${ }^{1}$, Leo Zhang ${ }^{2}$, and Enrico de Angelis ${ }^{1}$ \\ ${ }^{1}$ Politecnico di Milano, Milano, Italy \\ ${ }^{2}$ Trimble China, Beijing, China
}

\begin{abstract}
Evaluating building performances, such as the indoor daylight, is usually based on numerical simulation and $2 \mathrm{D}$ image illustration. It does not take advantage of emerging Augmented Reality (AR) or Mixed Reality (MR) techniques to intuitively aid the building design. The current study probes into the topic bringing building geometry information together with simulation results into $\mathrm{AR} / \mathrm{MR}$ environments. After introducing some precedent work in the literature, authors recap the typical workflow and report its application on two case studies. The first case study displays the structural simulation of a high-rise building in AR, while the second case study shows the daylight simulation of a two-story house within MR. At last, reflections are conducted and future possibilities are identified. Authors deem that, in the aim of this study, only retrofitting projects are suitable for MR. The present work can help practitioners better understand this new approach, offering the added value of using AR/MR for building design.
\end{abstract}

\section{Introduction}

Virtual Reality (VR), Augmented Reality (AR), and Mixed Reality (MR) are advanced technologies in interactions among people, computer, and reality. By allowing digital and physical objects coexist and providing an intuitive sense of how it might feel like to live in the animated environment, they become powerful tools for building designers. There are no unanimous and clear taxonomies of these virtual environments in the literature or in the industry (Flavián et al. 2018). For the present study, AR refers to technique overlaying virtual objects on the realworld environment while MR refers to techniques not just overlaying but anchoring virtual objects to the real world. The AR can be used indoor or outdoor while the MR seldom can be utilized outside a building.

Actually, it is not novel to aid the building design process with these virtual environments. As early as the 1990s, Donath \& Regenbrecht (1995) have started integrating VR with the architecture design. Afterwards, Broll et al. (2004) and Postema (2005) explored AR usages during the conceptual design stage. Following them, scholars integrated Building Information Modelling (BIM) with AR for interactive visualization (Belcher \& Johnson, 2008b; J. Wang, Wang, Shou, \& Xu, 2014; L. Wang, 2017). Moreover, educators found that AR also can improve the teaching quality of building design (Ayer et al., 2016; X. Wang, 2007; Milovanovic et al., 2017).

Although there have been considerable interests in interpreting building design in virtual environments, investigations on visualizing the performance simulation together in $\mathrm{AR} / \mathrm{MR}$ are still insufficient. Interrogatively visualizing these data in the $\mathrm{AR} / \mathrm{MR}$ can decrease misinterpretations and help designers make correct decisions ( $\mathrm{Li}$ et al. 2017). This topic was firstly coined by Belcher \& Johnson (2008a) who superimposed the virtual model with light simulation data obtained from software Radiance and Ecotect. Lakaemper \& Malkawi (2009) integrated the robot mapping, computational fluid dynamics (CFD), and $\mathrm{AR}$ to visualize the indoor thermal performance. Similarly, CFD and AR were integrated to efficiently diagnose the energy performance deviation in existing buildings (Golparvar-Fard \& Ham 2014). However, due to the fast development of AR/MR hardware and software, these studies in references may not reflect the up-to-date situation.

In the following part of this paper, the latest methodology and workflow of integrating building performance simulation within AR/MR will be introduced. Due to the scope of this work, specific simulation techniques and how simulation influences the decision-making in designs will not be expanded. Afterwards, two case studies dealing with different performance disciplines are reported. In the end, potentials and possibilities of this kind of $A R / M R$ usage will be explored.

The present work offers the latest knowledge to practitioners and inspires them to envisage other similar utilisations. Meanwhile, in the discussion section, authors point out appropriate applying scenarios of AR and MR respectively.

\section{Methodology}

\section{Apparatus}

It is exceptionally available and simple to practice AR because it requires no other equipment besides a regular tablet or smartphone. The device screen is the tangible interface to exhibit, add, size, and rotate 3D models. MR is higher demanding on the equipment than the AR. Windows 10 based HoloLens, developed by Microsoft, is the most advanced equipment in the 
current market (Kress \& Cummings 2017). It is the first self-contained holographic computer which does not need a PC or phone connection, nor external cameras (Microsoft 2018). HoloLens 2 is the latest version, which was issued on February 2019. Besides the HoloLens, Magic Leap One which was issued in the August 2018 is the only equipment has similar functions. One AR equipment can easily support several users observing the design together while multi-devices are required to hold up multi-users' simultaneous observation.

\section{Software}

Software used during the implementation process can be categorised into geometry modelling tools (Rhino, Revit, Sketchup, etc.), simulation tools (for different disciplines), format-conversion tools, and AR/MR Apps. The building geometry modelling and the simulation are not going to be discussed herein due to they are mature topics with affluent research papers. The mid-process software is similar between AR and MR.

Exporting building models into immersive virtual environments used to be time-consuming, let alone integrating simulation results together. However, it turns out to be much easier with the gaming engine Unity and others like Blender XR. For the final AR/MR Apps, ARki, Pair, and SmartReality are the best AR Apps elected by the architects' favourite website Archidaily (Grozdanic 2017). There are also many commercial MR Apps, like ARchitect and
SketchUp Viewer, etc. Utilizing Unity and Visual Studio is the sweeping workflow for making holographic Apps for the HoloLens (Hockett \& Ingleby 2016). At the same time, MR Apps also can be built up just by using the HoloLens emulator, without a HoloLens.

\section{Dataflow}

The present study will not deeply introduce functions or characteristics of individual software but focusing on the general dataflow and the data format conversion (Figure 1). Geometry modelling tools play two primary roles in the whole process. On one side, the geometry information is saved as a file in the MotionBuilder format (FBX) which enable all software to share these $3 \mathrm{D}$ data for the final presentation. On the other side, they also need to export the geometry information to simulators for simulation, via formats of IDF/IFC/gbXML. With the help of software like Blender, Paraview, and Unity, simulation results can be transformed into vector graphic formats, which can be integrated with building geometry and imported into the AR Apps. The last step is visualizing these data by Apps for $A R / M R$ equipment. Once the file is carried into these Apps, designers can scan in the paper or physical model version of building layouts and then interpret the design in AR/MR. Detailed dataflow is shown in Figure 1 and practical work is outlined in the following section with two case studies for AR and MR respectively.

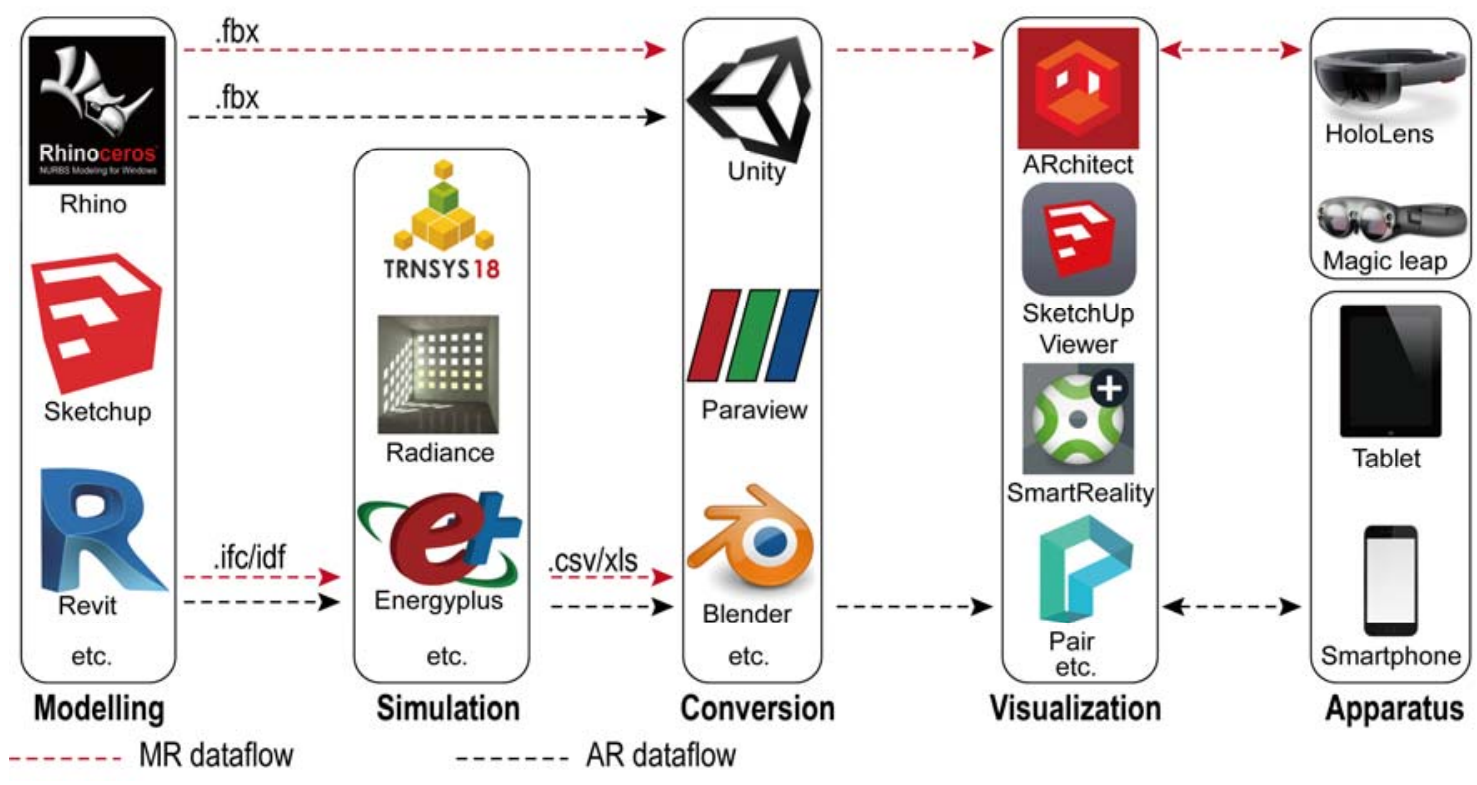

Figure 1: Dataflow of the whole process

\section{Case studies}

\section{The AR case}

Located in the Central Business District of Beijing, the 528-meter-tall skyscraper China Zun (Figure 2a) is the world's tallest building in a high seismic risk zone. Therefore, its structural design is deliberately based on a multi-objective optimization, aiming to obtain the max lateral stiffness, the least material cost, the minimum distance between mega columns and curtain 
walls. Moreover, there are aesthetical requirements from architects as well as various constraints demanded by Chinese building design codes, such as minimum member thickness, vertical and lateral load related column axial compression ratio. Therefore, good visualization of such performance-based results (Figure 2b) would be necessary for people to understand and improve their design work.

The structural performance is analysed by the software GSA, developed by the design company Arup. The GSA outputs structure members vs strain energy density, under a specified lateral force load case. The higher the strain energy density of a structural member, the more this structural member contribute to the lateral stiffness of the whole building structure. Overall lateral stiffness efficiency is significantly influenced by sizes and strain energy densities of structural members. The detailed building design process will not be discussed herein but more introductions on this design can be found in the previous publication (Cheng et al. 2016).

The workflow of visualizing strain energy density in 3D structural members are as following (Figure 2C):

1. Simulation results of strain energy density are obtained from the GSA, and then imported them into Rhino/Grasshopper as table arrays via the CSV format.

2. In the Grasshopper, values of strain energy density are designated with colours based on a colour contour range, and the colour information is assigned to each structure member of the original colourless geometry model.

3. The colour-assigned structural model is exported as a file in the FBX format.

4. The FBX file is imported to a pre-defined Unity file. In the Unity, the colour assigned model is rescaled and positioned in relation to the $2 \mathrm{D}$ plan of China Zun, which will act as the signature marker for the AR App to recognise and to position the model in the AR environment.

5. By using the XCode, designers export the Unity file as an IPA file which could be installed to iPhone or iPad via iTunes. The IPA file is opened by the Arup Real, an in-house AR App developed by Arup and based on the Vuforia engine. This App is only for the IOS system so that designers can see the design on an iPhone or iPad.

6. Designers capture the printed floor plan in the camera view by using Arup Real on an iPhone or iPad. After that, the building model with the colour contour of strain energy density could be visualized in AR.

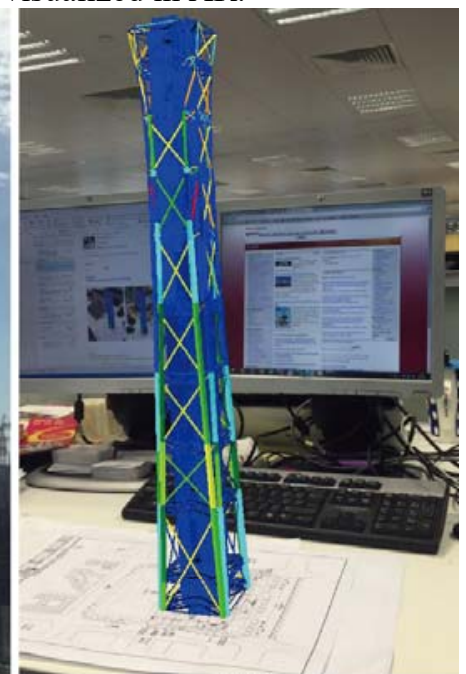

(b)

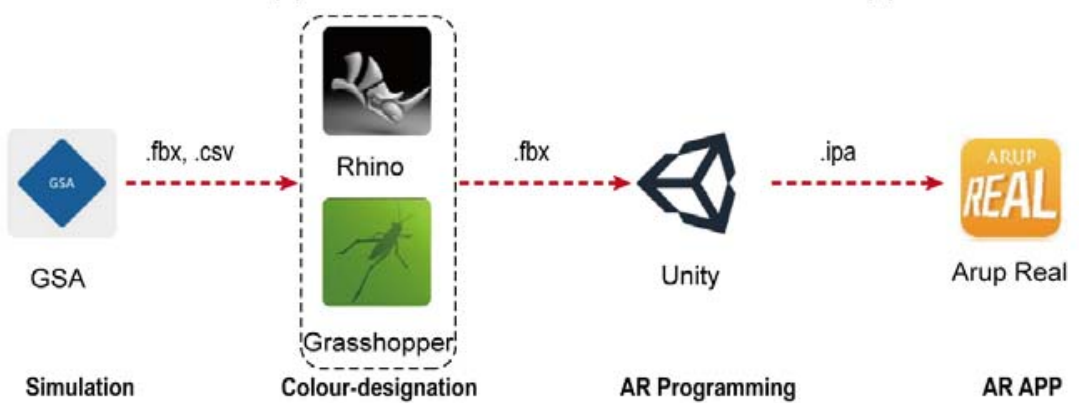

(c)

Figure 2: AR visualization of China Zun (a, China Zun in AR and in the physical reality; $b$, a screenshot of Strain Energy Density colour contour; $c$, dataflow of the process) 


\section{The MR case}

The second case is using MR to visualize a simple house design with illuminance simulation results. The house is located in the city of Cairo which has a climate with extreme solar heat. Radiation is too intense to be ignored when seeking for the interior daylight illuminance in Cairo. Therefore, this project has a focal point balancing the indoor visual comfort and the thermal radiation from daylight. The initial building geometry is built in the Autodesk Formit and roughly analysed with the cloud-based simulation software Insight 360 . Then the sketchy geometry is imported in the Autodesk Revit for a deeper design, during which more shading devices are put on facades. The detailed design process will not be introduced herein because it is not the focal point. After the model is completely built, the daylight is simulated by the Green building studio, cloud-based simulation software embedded in the Autodesk Revit. The analysis plane height is set at $80 \mathrm{~cm}$ while the June 22nd (summer solstice) and December 22nd (winter

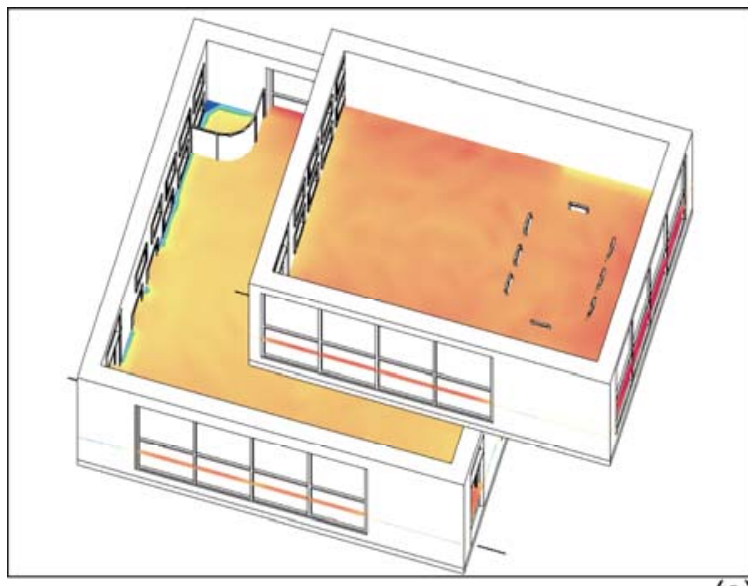

(a)

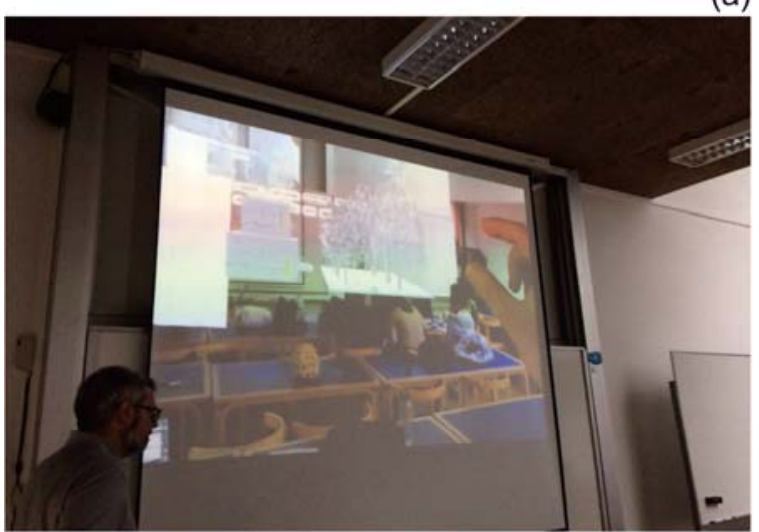

(c) solstice) are selected for the simulation because they are two extreme dates. The brighter colour in simulation results (Figure 3a) represents the higher illuminance reached inside the house.

Afterwards, it is the data processing stage which already has been introduced in chapter 2.2. Building geometry information is exported as an Autodesk MotionBuilder format (FBX) file, BIM data (such as window size) and simulation results are exported as excel files. Simulation result at each point is represented as a 3D box with colour (Figure $3 d$ ) rather than a $2 \mathrm{D}$ textured representation. In the end, the geometrical model, the model element data (BIM data), and illuminance simulation results are combined in the program Unity $3 \mathrm{D}$ and exported to an App for visualizing the data in the HoloLens. The final interactive App herein is developed by Middle East Technical University. It provides interactions with the model in different scales. Users can navigate freely inside the building, and they can rotate, scale, and move objects, by gesturing and pointing (Figure $3 b$ ).

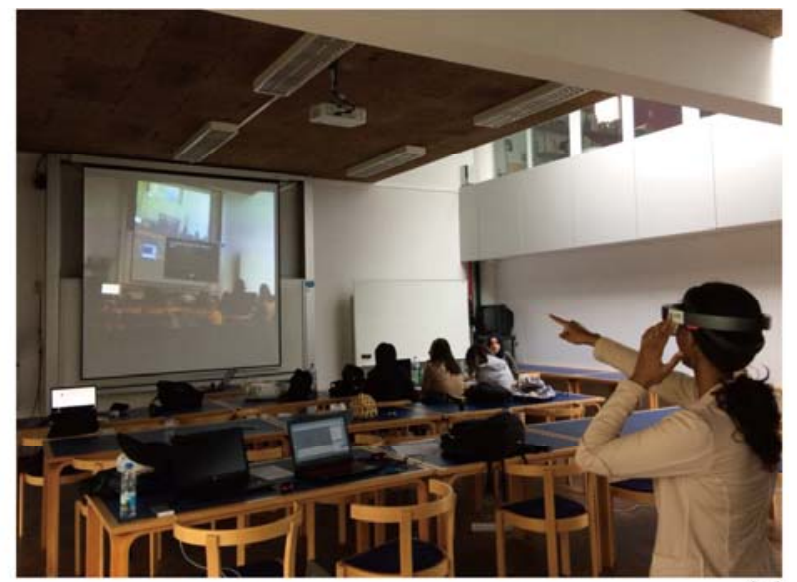

(b)

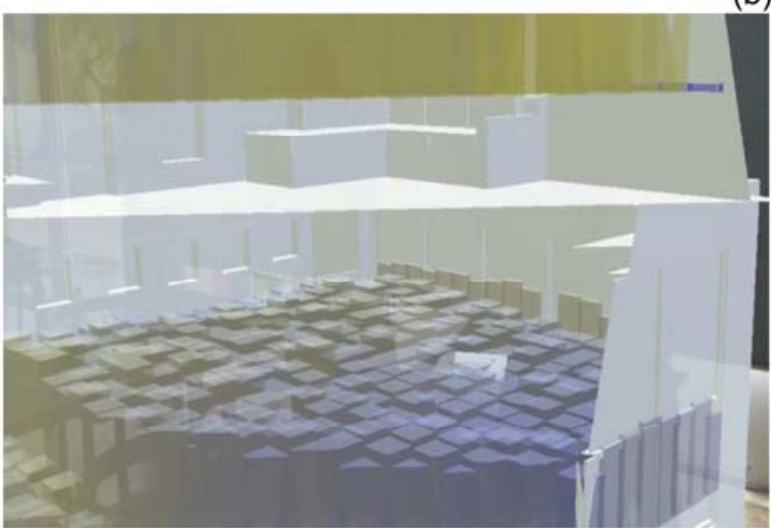

(d)

Figure 3: The second study (a, geometry model with the simulation result in Revit; $b$, the working site and monitoring screen; $c$, operating the model with gestures; $d$, a snapshot of simulation results viewed in HoloLens)

\section{Discussions}

\section{Reflections}

Visualizing building geometries together with simulation results in both AR and MR helps designers make better-informed decisions. The core of the present topic is $\mathrm{AR} / \mathrm{MR}$ enabling visualization of invisible concepts or events by superimposing virtual objects or information onto physical objects or environments (Mekni \& Lemieux 2014). Therefore, the current topic can be discussed from three aspects: invisible concepts (which category of building performance evaluation), virtual objects or 
information (how to visualize invisible concepts), physical objects or environments (physical bases to integrate virtual objects).

Firstly, invisible concepts (building performance objectives) aimed for both AR and MR should be spatially based and place-varying, otherwise there is no necessity exhibiting them in virtual environments whose goodness is to improve the spatial perception. For example, a building's total energy consumption is easy to be understood in a 2D chart. However, if we transform the indicator into energy consumption intensity (thermal zone varied), it would be more suitable for the current topic.

The second issue is to transform the invisible simulation information into perceivable objects, such as $3 \mathrm{D}$ charts and heat maps, etc. Appropriate selection can make people less confused when facing the complex data. For example, in the second case study, interpreting the simulation results by $3 \mathrm{D}$ boxes can let people easily understand the overall condition in a room. Another advantage of visualization in AR/MR is the ability to identify performance variations on the time dimension, which is especially useful for noninstantaneous simulations, such as the wind movement.

At last, for the physical objects and environments holding the virtual model, using a printed floor plan or a physical model by $3 \mathrm{D}$ printing are two main ways for the AR. Interpreting new designs in MR, as the second case in the present paper, actually does not fully utilize MR's advancements because virtual objects do not have any practical correlations with the real physical surroundings. The same intention can be easily achieved by just using the VR or AR. From another perspective, only retrofitting projects which has the original construction framework to impose virtual objects may be more suitable to use the MR

\section{Future possibilities}

There are four future opportunities deserve to be mentioned herein. First of all, the current performance simulation and AR/MR visualization are not linked. Both case studies need manually inputting rather than automatically updating simulation results after design modifications. Future platforms under the present topic should also enable people to stream data in real time or designing high-performance buildings in the immersive virtual world (Sgambelluri 2017), such as the App vSpline allowing users to intuitively create, manipulate, analyze, and 3D print physical prototypes all within VR (vSpline 2018). After designers modifying building models in virtual environments directly, simulation results can be demonstrated on the virtual model in immediately.

The second development orientation is combining AR/MR with the performance-based generative design. By integrating parametric modelling, simulation, and optimization, there will be hundreds of design solutions automatically generated. Detailed information of this design method can be found in another literature review (Zhao \& Angelis n.d.). Together with their respective performance simulation results, all of them can be exhibited and navigated in web-based applications like DesignExplorer (Thornton 2017) or Project Fractal (Autodesk 2017). With the technique WebXR (W3C 2018), these applications can be straightforwardly realized in virtual environments. It is more easily than the previous idea modifying design solutions in AR/MR directly.

The third opportunity is to develop an integrated platform incorporating fragmental information from different disciplines, allowing users to seamlessly switch among them. For example, one layer of daylight simulation result and another layer of thermal comfort simulation (of different thermal zones) shown on the same building floor. When people switch between them, one of them can be visible while the other becomes hidden. Such kind of integrative and interactive representation offers a comprehensive understanding of building performance. However, the reaction speed of that model may slow down when it carries too much information.

The last possibility is using the tangible media techniques to bring the design and simulation information back into the real world. By representing them in the form of pixels on bit-mapped displays, tangible media techniques can bring information from the virtual environments to the reality (Ishii 2008). So, it can be integrated into the design workflow in the present study if a more intuitive feeling is required by architects or building designers (Figure 4). A related experiment can be found in the study conducted by Fitzgerald \& Ishill (2018).

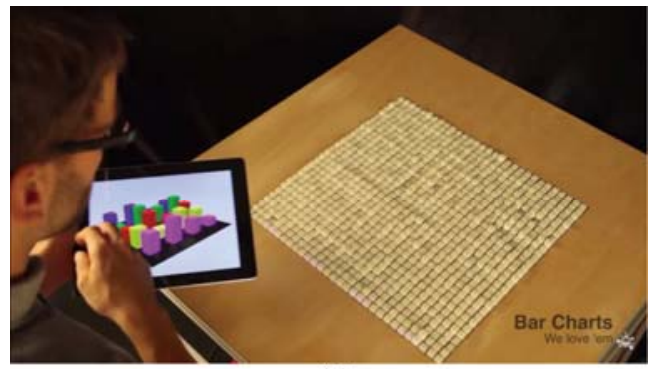

(a)

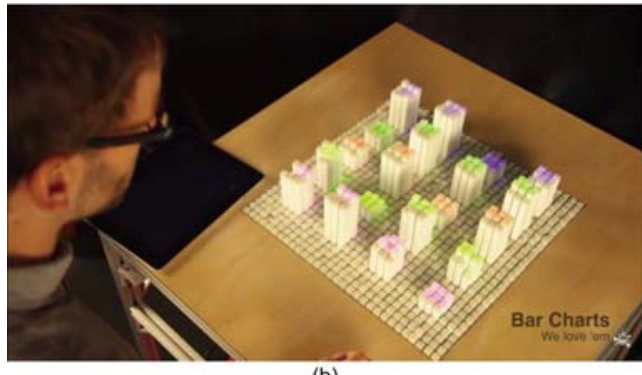

(b)

Figure 4: Tangible Media Techniques (a, geometry and colour information in a tablet; $b$, these information was represented in the real world. (Leithinger et al. 2013)) 


\section{Conclusions}

The present study recaps the general process of visualizing building geometries together with performance simulation results in AR/MR environments. Demonstrating the spatiotemporal building performance more intuitively, this type of design interpretation can help people avoid errorprone decision makings. Two practical case studies are reported, representing AR and MR respectively. In the end, these AR/MR usages are provokingly reflected on and future research opportunities are also discussed. Authors pointed out that projects designing new buildings, other than building retrofitting, may have limited meaning showing simulation results together in the MR. In that case, using MR has no apparent advantage than AR or VR. Their respective suitability for various applying scenarios will be deeply distinguished in future work.

\section{Acknowledgments}

Other team members contributed on the design project of AR case and thanks to the other participators of the MR case workshop.

\section{References}

Autodesk. (2017) Project Fractal. Project Fractal. Available at: https://home.fractal.live/ [Accessed November 12, 2018].

Ayer, S.K. et al. (2016) Augmented Reality Gaming in Sustainable Design Education. Journal of Architectural Engineering, 22(1), pp.1-9.

Belcher, D. \& Johnson, B. (2008). MxR A physical model-based mixed reality interface for design collaboration, simulation, visualization and form generation. In ACADIA 08: Silicon + Skin: Biological Processes and Computation: Proceedings of the 28th Annual Conference of the Association for Computer Aided Design in Architecture. pp. 464-471. Available at: http://www.scopus.com/inward/record.url?eid=2 -s2.0-84858836626\&partnerID=tZOtx3y1.

Belcher, D. \& Johnson, B.R. (2008) ARchitecture View An Augmented Reality Interface for Viewing 3D Building Information Models. In Architecture in Computro, 26th eCAADe Conference Proceedings. pp. 561-568. Available at: http://dmg.caup.washington.edu/.

Broll, W. et al. (2004) ARTHUR: A Collaborative Augmented Environment for Architectural Design and Urban Planning. Journal of Virtual Reality and Broadcasting, 1(1), pp.1-10.

Cheng, Y., Citerne, D. \& Zhang, L. (2016) The Integrated Smart Design Technologies for Tall Building Structural Design | 集成化智能设计技 术在超高层结构设计中的应用. In $C T B U H$
2016. Shenzhen, pp. $705-712$.

Donath, D. \& Regenbrecht, H. (1995) VRAD (Virtual Reality Aided Design) in the Early Phases of the Architectural Design Process. In Sixth International Conference on Computer-Aided Architectural Design Futures. p. 779. Available at: $\quad$ http://papers.cumincad.org/cgibin/works/paper/8955.

Fitzgerald, D. \& Ishill, H. (2018) Mediate : A Spatial Tangible Interface for Mixed Reality. In CHI 2018. Montréal, pp. 1-6.

Flavián, C., Ibáñez-Sánchez, S. \& Orús, C. (2018) The impact of virtual, augmented and mixed reality technologies on the customer experience. Journal of Business Research, (January), pp.114. Available at: https://doi.org/10.1016/j.jbusres.2018.10.050.

Golparvar-Fard, M. \& Ham, Y. (2014) Automated Diagnostics and Visualization of Potential Energy Performance Problems in Existing Buildings Using Energy Performance Augmented Reality Models. Journal of Computing in Civil Engineering, 28(January), pp.17-29. Available at: http://ascelibrary.org/doi/abs/10.1061/(ASCE)C P.1943-5487.0000311.

Grozdanic, L. (2017) The Top 5 Virtual Reality and Augmented Reality Apps for Architects. ArchDaily. Available at: https://www.archdaily.com/878408/the-top-5virtual-reality-and-augmented-reality-apps-forarchitects.

Hockett, P. \& Ingleby, T. (2016) Augmented Reality with HoloLens: Experiential Architectures Embedded in the Real World, Available at: http://arxiv.org/abs/1610.04281.

Ishii, H. (2008) The tangible user interface and its evolution. Communications of the ACM, 51(6), pp.32-36. Available at: http://portal.acm.org/citation.cfm?doid=134902 6.1349034 .

Kress, B.C. \& Cummings, W.J. (2017) Towards the ultimate mixed reality experience: HoloLens display architecture choices. In Digest of Technical Papers - SID International Symposium. pp. 127-131.

Lakaemper, R. \& Malkawi, A.M. (2009) Integrating Robot Mapping and Augmented Building Simulation. Journal of Computing in Civil Engineering, 23(6), pp.384-390. Available at: http://www.scopus.com/inward/record.url?eid=2 -s2.0-70350075850\&partnerID=tZOtx3y1.

Leithinger, D. et al. (2013) inFORM. Tangible Media Group. Available at: https://tangible.media.mit.edu/project/inform/ 
[Accessed December 24, 2018].

Li, W., Nee, A. \& Ong, S. (2017) A State-of-the-Art Review of Augmented Reality in Engineering Analysis and Simulation. Multimodal Technologies and Interaction, 1(3), p.17. Available at: http://www.mdpi.com/24144088/1/3/17.

Mekni, M. \& Lemieux, A. (2014) Augmented Reality : Applications, Challenges and Future Trends. In 13th International Conference on Applied computer and applied computational science. pp. 205-214.

Microsoft. (2018) Microsoft HoloLens. Available at: https://www.microsoft.com/en-us/HoloLens [Accessed December 12, 2018].

Milovanovic, J. et al. (2017) Virtual and Augmented Reality in Architectural Design and Education To cite this version : HAL Id : hal-01586746 An Immersive Multimodal Platform to Support. In CAADFutures 17. pp. 513-532.

Postema, J.J. (2005) The use of Mixed Reality in architecture for conceptual design. In 2nd Twente Student Conference on IT. pp. 1-7.

Sgambelluri, M. (2017) Next-Gen Virtual Reality Will Let You Create From Scratch-Right Inside VR. Available at: https://www.autodesk.com/redshift/createvirtual-reality/.

Thornton, T. (2017) Design Explorer 2. Github. Available at: http://ttacm.github.io/DesignExplorer/.

vSpline. (2018) About vSpline. Available at: http://www.vspline.com/features.html [Accessed December 17, 2018].

W3C (2018) WebXR Device API. Available at: https://immersive-web.github.io/webxr/ [Accessed December 17, 2018].

Wang, J. et al. (2014) Integrating BIM and augmented reality for interactive architectural visualisation. Construction Innovation, 14(4), pp.453-476.

Wang, L. (2017) Augmented Reality Visualization of Building Information Model. The Ohio State University.

Wang, X. (2007) Augmented reality potentials in design curriculum. Engineering and Technology, 6(1), pp.119-122.

Zhao, S. \& Angelis, E. de (in press). Performancebased Generative Architecture Design: A Review on Design Problem Formulation and Software Utilization. Journal of Integrated Design and Process Science. Available at: https://content.iospress.com/articles/journal-ofintegrated-design-and-processscience/jid190001. 